Varying the Intensity of Competition in a Multiple Prize Rent Seeking Experiment

\author{
Lisa R. Anderson \\ College of William and Mary \\ Beth A. Freeborn \\ College of William and Mary
}

College of William and Mary

Department of Economics

Working Paper Number 75

August 2008 
COLLEGE OF WILLIAM AND MARY

DEPARTMENT OF ECONOMICS

WORKING PAPER \# 75

August 2008

\title{
Varying the Intensity of Competition in a Multiple Prize Rent Seeking Experiment
}

\begin{abstract}
We experimentally test a rent seeking model under five levels of competition. At one extreme, a subject's probability of winning a prize is equal to her share of the total expenditures. At lower levels of competition, a subject's probability of winning is affected more by her own expenditures than by the expenditures of others. Predicted expenditure levels are positively associated with higher levels of competition. Consistent with previous rent seeking experiments, we find that subjects spend significantly more than the Nash equilibrium prediction at all levels of competition. However, expenditure patterns generally follow the Nash prediction; expenditures decrease as the level of competition decreases. Our experimental design also includes a lottery choice experiment to control for subjects' risk preference. We find that subjects who are more risk averse spend significantly less in the contest and this effect is particularly strong for female subjects.
\end{abstract}

JEL Codes: C91, D72

Keywords: rent seeking, experiment, rent dissipation, political competition

Lisa R. Anderson

Department of Economics

College of William and Mary

Williamsburg, VA 23187-8795

lisa.anderson@wm.edu
Beth A. Freeborn

Department of Economics

College of William and Mary

Williamsburg, VA 23187-8795

bafree@wm.edu 


\section{Introduction}

Standard examples of rent seeking contests include competition to become a monopoly, interest groups seeking benefits from a regulator, or firms looking to be awarded government contracts. The large theoretical literature has motivated a small but growing experimental literature on rent seeking. The consensus from experimental investigations of Tullock's (1980) classic rent seeking model is that subjects overspend relative to the Nash equilibrium prediction (e.g., Millner and Pratt, 1989 and 1991). This result holds in more recent extensions that include entry costs and heterogeneity (Anderson and Stafford, 2003), risk in the value of the rent (Öncüler and Croson, 2005), multi-stage contests (Cadigan, 2007), and endogenous determination of the value of the rent (Bullock and Rutström, 2007).

These experimental studies of rent seeking have adhered to an important assumption of Tullock's (1980) model; competitors vie for a single prize. Many policy decisions, however, concern the allocation of more than one prize. For example, a typical policy maker is likely to be lobbied by many groups that are not necessarily competing for the same single prize. The lobbying efforts are not totally unrelated, since policy makers face budget constraints. Tighter budget constraints give rise to more competition in the sense that lobbying efforts by one group have a bigger effect on the other group's likelihood of success. This is the rent seeking aspect of the model.

We follow Godwin et al. (2006) in considering a rent seeking contest that incorporates a competition parameter; this parameter alters the standard model such that multiple prizes may be awarded. ${ }^{1}$ At the most competitive extreme, an individual's probability of winning a prize is equal to her share of the total expenditures, as in the standard rent seeking model. At lower levels

\footnotetext{
${ }^{1}$ Other theoretical studies that model rent seeking contests with the possibility of more than one prize include Clark and Riis (1996, 1998), Berry (1993) and Yates and Heckelman (2001).
} 
of competition, an individual's probability of winning a prize is affected more by her own expenditures than by the expenditures of others. In this case, a win by one individual does not rule out a win by the other individual, but it does decrease the other person's probability of winning.

One compelling feature of the model is that relaxing competition reduces predicted rent seeking expenditures relative to the standard model. Godwin et al. (2006) note that these predictions might reflect more realistic estimates of actual expenditures, since the standard model typically predicts total rent seeking expenditures that exceed the value of the rent. We test these predictions experimentally using a subject pool of one hundred and forty undergraduate students. Consistent with previous rent seeking experiments, we find that subjects spend significantly more than the Nash equilibrium prediction at all levels of competition. However, expenditure patterns generally follow the Nash prediction in the sense that they decrease as the level of competition decreases. Our design also includes a lottery choice experiment to control for subjects' risk preference. We find that subjects who are more risk averse spend significantly less in the contest and this effect is particularly strong for female subjects.

The next two sections provide additional details about the rent seeking model and the experimental design. Section 4 presents results and section 5 concludes.

\section{Model}

This section presents a modified version of the model from Godwin et al. (2006), which is an extension of Tullock's (1980) classic rent seeking model. Two agents compete for a prize of value $V$. The likelihood of winning the prize is a function of the agent's own expenditure level, $\left(E_{i}\right)$, and the expenditure level of the other agent, $\left(E_{j}\right)$. The model also includes a 
competition parameter $(0<\alpha \leq 1)$, which measures the effect of player $j$ 's expenditures on player i's probability of winning the prize. The probabilities of winning (and not winning) the prize $V$ are expressed as:

$$
\begin{gathered}
P_{t}=\left[\frac{E_{t}}{E_{t}+\sigma E_{f}}\right]=\text { probabllity that agent } 1 \text { wins the prize } \\
1-P_{t}=\left[\frac{\omega E_{j}}{E_{i}+\sigma E_{f}}\right]=\text { probability that agent } 1 \text { does not win the prize. }
\end{gathered}
$$

Note that in the extreme case of competition $(\alpha=1)$, the sum of the two agents' probabilities of winning the prize is equal to one. Thus, any increase in one agent's probability of success is exactly offset by a decrease in the other agent's probability of winning the prize. At lower levels of competition, the expenditure of one agent has a smaller impact on the likelihood of the other agent winning the prize. Another feature of this variation on the classic model is the assumption that there can be more than one prize. Specifically, for $\alpha<1$, there are four possible outcomes: (1) both players win the prize; (2) player $i$ wins and player $j$ does not win the prize; (3) player $i$ does not win and player $j$ wins the prize; (4) neither player wins the prize.

Given the probability function above, each agent maximizes expected profits, the difference between expected returns and expenditures, as follows:

$$
\mathbf{E}\left[\pi_{i}\right]=\left\lceil\frac{E_{i}}{E_{t}+a E_{j}}\right\rceil \gamma-E_{i}
$$

with $0<\alpha \leq 1$. The first order condition is given by

$$
\frac{d w_{t}}{E_{t}}=\left[\frac{\omega E_{j}}{\left(E_{t}+\alpha E_{f}\right)^{2}}\right] V-1=0
$$

which results in a best response function 


$$
\rho_{t}=\left\{\begin{array}{cc}
\sqrt{\alpha E_{f} V}-\alpha E_{f y} & 0<E_{f} \alpha_{h} \frac{V}{\alpha} \\
0_{r} & E_{l}>\frac{V}{\alpha}
\end{array} .\right.
$$

Thus, the symmetric equilibrium expenditure level is

$$
\rho_{\varepsilon}=\frac{\alpha \vartheta}{(1+\alpha)^{2}}
$$

and equilibrium payoffs are

$$
\pi f=\frac{\rho_{s}}{\rho_{s}+\alpha \rho_{s}} V-\rho_{f}
$$

In addition to the standard comparison of observed behavior and the Nash prediction, an additional testable implication of this model is that equilibrium expenditures are increasing in $\alpha$; as competition increases, agents increase their expenditures and as competition decreases, agents decrease their expenditures level.

\section{Experimental Design}

One hundred and forty undergraduate students at the College of William and Mary participated in the experiment. Subjects were given the instructions handout in Appendix A, which was also read aloud. There were two bidders, A and B, in each contest, and the probability of person $A$ winning a prize was $E_{A} /\left(E_{A}+\alpha E_{B}\right)$ where $E_{i}$ denotes the effort level of person $i$ and $\alpha$ is the competition parameter. In each contest subjects were given $\$ 10$ to spend on units of effort, which could be purchased in penny increments. Thus, the effort level was identical to the expenditure level and ranged from 0 to 1000. Tullock's (1980) model is represented when $\alpha$ is 1 . In addition to the baseline of $\alpha=1$, we considered values of $\alpha$ equal to $0.5,0.25,0.1$ and 0.05 . Each value of $\alpha$ corresponded to a different scenario number in the instructions, and subjects were given Excel spreadsheets for each scenario that calculated their 
probability of winning the prize for any combination of effort levels they entered. These spreadsheets are displayed in Appendix B. Subjects made decisions for all scenarios without receiving any feedback about the other player's choices. Also, subjects were told they could make decisions in any order they chose, thus minimizing any possible order effects. ${ }^{2}$ Once all decisions were made, the record sheets were collected and one scenario was randomly selected for payment by the throw of a 6 -sided die.

We used 100 poker chips to randomly determine whether or not subjects won the prize. Each chip had an orange number written on one side and a blue number written on the other side. The orange number and the blue number on each chip summed to 100. Scenario 1 corresponded to the baseline treatment of $\alpha=1$ with the assumption that only one prize was available. If this scenario was chosen for payment, we awarded the prize by drawing one poker chip from an opaque container. If the orange number on the chip was less than or equal to the orange player's probability of winning in the chosen scenario, the orange player was awarded the $\$ 10$ prize. Alternatively, if the blue number on the chip was less than or equal to the blue player's probability of winning, the blue player was awarded the $\$ 10$ prize. $^{3}$

As noted above, a novel feature of the rent seeking model used here is the assumption that more than one prize is available for any $\alpha<1$. To implement scenarios with more than one possible prize, we used the same set of 100 poker chips labeled with orange and blue numbers. We first drew a poker chip for the orange player. If the orange number on the chip was less than or equal to the orange player's probability of winning in the chosen scenario, the orange player

\footnotetext{
${ }^{2}$ Most subjects were observed to be skipping around in the set of scenarios rather than moving down the list in the order presented on the decision sheet.

3 As noted in the instructions, in the case of a tie, our procedures called for us to flip the poker chip to determine the winner. Ties were possible in scenario 1 if the blue and orange numbers on the chip drawn were equal to the probabilities of winning for the blue and orange players. Scenario 1 was randomly selected for payment in only one session and there were no ties.
} 
was awarded a $\$ 10$ prize. After returning the chip to the container, we next drew a chip for the blue player. If the blue number on the chip was less than or equal to the blue player's probability of winning, the blue player was awarded a $\$ 10$ prize. Note that this selection method allowed for zero, one or two prizes to be awarded.

To explore whether the method of awarding the prize (drawing one chip versus two chips) had an effect on behavior in the experiment, we included scenario 2 with $\alpha=1$ but with more than one prize available (i.e., using the two chip method to award prizes). Note that the calculation of probabilities is exactly the same across these two treatments. For any combination of expenditure levels by the two players, the probabilities of winning and the Nash prediction in scenario 1 is exactly the same in scenario 2 . Thus, we can compare the observed behavior in scenario 1 and scenario 2 to test whether the method of awarding prizes affected behavior, conditional on the same probability of winning. The additional scenarios ( 3 through 6 ) represented values of $\alpha$ equal to $0.5,0.25,0.1$ and 0.05 , respectively. The experiment lasted about 45 minutes and subjects earned an average of $\$ 12.16$, in addition to a show up payment of $\$ 5$.

\section{Results}

We first examine the effect of the competition parameter, $\alpha$, on individual effort and participation in the contest (i.e., choosing a non-zero effort level). Table 1 presents the Nash predicted effort level and average observed effort for the six scenarios. Values of $\alpha$ were chosen such that the Nash predicted effort choice varied greatly across scenarios. ${ }^{4}$ Consistent with previous studies, the mean observed effort exceeds the Nash prediction in every scenario. The

\footnotetext{
${ }^{4}$ The Nash effort level decreases dramatically between scenarios 3 and $4(28 \%), 4$ and 5 (48\%), and 5 and 6 (46\%). The difference in Nash effort level for Scenarios 2 and 3, however, is relatively small (11.1\%)
} 
fifth column provides the t-statistic for the test that the mean effort level is equal to the Nash predicted effort level. In each scenario, the observed effort is significantly greater than the Nash prediction at the $1 \%$ level. The actual participation rate was somewhat low, given the Nash prediction that every subject should choose a positive effort level. However, the percentage of people who made a non-zero effort choice generally increased as $\alpha$ fell. Recall that as $\alpha$ decreases, the effort choice of the other player has a smaller effect on the probability of the subject winning a prize, and for any given combination of effort levels the probability of winning increases. Thus, while not predicted by theory, it appears that lower levels of competition draw more people into the contest.

Table 1: Summary Statistics

\begin{tabular}{lcccccc}
\hline \hline & & $\begin{array}{c}\text { Nash } \\
\text { Effort } \\
\text { Level }\end{array}$ & $\begin{array}{c}\text { Number } \\
\text { of Prizes } \\
\text { Possible }\end{array}$ & $\begin{array}{c}\text { Mean } \\
\text { Effort } \\
\text { Level }\end{array}$ & t-statistic & $\begin{array}{c}\text { Participation } \\
\text { Rate (\%) }\end{array}$ \\
\hline Scenario 1 & 1.0 & 250 & 1 & 414.6 & 6.811 & 89.3 \\
Scenario 2 & 1.0 & 250 & 0,1 or 2 & 445.0 & 7.797 & 90.7 \\
Scenario 3 & 0.5 & 222 & 0,1 or 2 & 444.0 & 10.33 & 95.0 \\
Scenario 4 & 0.25 & 160 & 0,1 or 2 & 409.8 & 12.38 & 97.9 \\
Scenario 5 & 0.10 & 83 & 0,1 or 2 & 349.4 & 12.06 & 98.6 \\
Scenario 6 & 0.05 & 45 & 0, 1 or 2 & 323.3 & 10.62 & 98.6 \\
\hline
\end{tabular}

Note: There are 140 observations for each scenario.

Table 2 displays relative comparisons of effort choice to test the prediction that effort declines with $\alpha$. The first column presents the Nash prediction on behavior and the second column reports the z-statistic from the Wilcoxon signed-rank test that the effort choices are equal. For the Nash prediction that effort choices are equal (first row), a significant z-statistic is evidence that the observed choices are not equal. For the Nash predictions that one effort choice is larger than the other, a positive and significant z-statistic confirms the Nash prediction (a negative and significant z-statistic implies the opposite of the Nash prediction). 
Table 2: Effort Choice Dynamics

\begin{tabular}{cc}
\hline \hline $\begin{array}{c}\text { Nash Prediction } \\
\text { for Pattern of Behavior }\end{array}$ & $\begin{array}{c}\text { Z-Statistic from } \\
\text { Wilcoxon Signed-Rank Test } \\
(\mathrm{p} \text {-value) }\end{array}$ \\
\hline Effort(1) $=$ Effort(2) & $-1.708^{*}$ \\
& $(0.09)$ \\
Effort(2) $>$ Effort(3) & -0.489 \\
& $(0.62)$ \\
Effort(3) $>$ Effort(4) & $3.122^{* * *}$ \\
& $(0.002)$ \\
Effort(4) $>$ Effort(5) & $5.367^{* * *}$ \\
& $(0.000)$ \\
Effort(5) $>$ Effort(6) & $3.996^{* * *}$ \\
& $(0.000)$ \\
\hline \hline
\end{tabular}

$*, * *$ and $* * *$ indicate significance at the $10 \%$ level, $5 \%$ level and $1 \%$ level, respectively.

Notice from the first row of Table 2 that the Nash prediction is that effort in scenario 1 is equal to effort in scenario 2 . Recall the only difference between the two decisions is that scenario 1 allowed for a single winner and scenario 2 allowed for multiple winners. The observed behavior, however, shows that effort in scenario 1 was smaller than effort in scenario 2, although only marginally significant $(\mathrm{p}=0.09)$. This result implies that the difference in the mechanism used to pick a winner had a small effect on behavior. It also suggests that researchers should use caution in comparing results across experimental studies when there are significant differences in procedures. Based on this finding, we use scenario 2 as the starting point to examine the effect of changing the competition parameter on effort choices.

The Nash prediction is that effort will be lower with each successive scenario, because effort choice is positively correlated with $\alpha$. Counter to theory, we do not find a significant difference in the observed effort choices of scenarios 2 and 3, but this is likely due to the fact that the Nash predicted effort levels are relatively close (250 and 222). The final three rows of Table 2 show that the observed behavior is generally consistent with the Nash prediction regarding 
patterns of behavior, and these results are significant at the $1 \%$ level. ${ }^{5}$ These findings provide some evidence that behavior is sensitive to the Nash prediction. To further study the relationship between the Nash prediction and actual choices in the experiment, we perform regression analysis. This analysis also incorporates subject-specific traits that are not included in the standard theory of rent seeking but are known to affect behavior in other experimental settings.

One subject-specific trait that has been demonstrated to affect behavior in experiments is risk aversion. Thus, in addition to the rent-seeking experiment, subjects also participated in a lottery choice experiment designed to elicit a measure of risk aversion (see Holt and Laury, 2002). The lottery choice experiment contained ten pairs of choices between a relatively safe lottery (with a payoff of $\$ 4.80$ or $\$ 6.00$ ) and a relatively risky lottery (with a payoff of $\$ 0.30$ or \$11.55) with different probabilities associated with the high and low payoffs for each of the choices. ${ }^{6}$ The probabilities were structured such that the expected payoff of the safe lottery exceeded that of the risky lottery for the first four decisions and the risky lottery had a higher expected payoff for the last six choices. Hence, a risk neutral subject will choose the safe lottery in the first four decisions and the risky lottery in the last six. Holt and Laury (2002) and Anderson and Mellor (forthcoming) provide additional details about how decisions in the lottery experiment are used to calculate a range of relative risk aversion for each subject. In the analysis that follows, we used the midpoint of the range of the coefficient of relative risk aversion (hereafter, mid-CRRA) for each subject to capture risk tolerance, and higher midpoints represent higher levels of risk aversion.

\footnotetext{
${ }^{5}$ Moving down the rows of Table 1, notice that actual effort did not fall in proportion to the predicted effort level. For example, comparing scenario 3 to scenario 4 , effort is predicted to be 28 percent lower in scenario 4 but is actually only 7.7 percent lower.

${ }^{6}$ The average payoff for the lottery choice experiment was $\$ 6.65$.
} 
Table 3 shows a summary of the results from the lottery choice experiment. In their survey of the experimental literature, Eckel and Grossman (forthcoming) present evidence that women are generally more risk averse than men, so Table 3 is separated by gender. Overall, $16 \%$ of the male subjects and $22 \%$ of the female subjects have a mid-CRRA within the range $(-0.15$ and 0.15$)$ of risk neutral behavior. The distribution of Table 3 shows that our subject pool is atypical in the sense that the female subjects were generally less risk averse than the male subjects. $^{7}$

Table 3: Distribution of Risk Aversion Parameter by Gender

\begin{tabular}{cccc}
\hline $\begin{array}{c}\text { Range of Relative Risk } \\
\text { Aversion }\end{array}$ & Risk Preference & \multicolumn{2}{c}{ Proportion of Choices } \\
r $<-0.95$ & Men & Women \\
\hline$-0.95<\mathrm{r}<-0.49$ & Risk Loving Range & 0.00 & 0.00 \\
$-0.49<\mathrm{r}<-0.15$ & & 0.00 & 0.02 \\
$-0.15<\mathrm{r}<0.15$ & Risk Neutral Range & 0.16 & 0.07 \\
\hline $0.15<\mathrm{r}<0.41$ & & 0.11 & 0.22 \\
$0.41<\mathrm{r}<0.68$ & Risk Averse Range & 0.34 & 0.03 \\
$0.68<\mathrm{r}<0.97$ & & 0.22 & 0.19 \\
$0.97<\mathrm{r}<1.37$ & & 0.07 & 0.06 \\
$1.37<\mathrm{r}$ & & 0.03 & 0.00 \\
\hline \hline
\end{tabular}

Konrad and Schlesinger (1997) show that risk aversion has an ambiguous theoretical effect on rent seeking expenditures; risk averse subjects may choose a very low effort level or choose not to participate so as to keep a large amount of their starting budget. On the other hand, the probability of winning the prize increases with effort, and so risk averse subjects may choose higher effort to increase the likelihood of winning the prize. The net effect of increasing risk

\footnotetext{
${ }^{7}$ In our subject pool, $53 \%$ of the subjects were male and $47 \%$ were female.
} 
aversion on expenditures depends on specific assumptions of the model. Hence, the effect of risk aversion on rent seeking is an open question which we address in the empirical analysis below. ${ }^{8}$

To analyze the determinants of individual effort levels, we perform linear regressions of actual effort on the Nash predicted effort level and other covariates. Results from these regressions are presented in Table 4. The unit of observation is the person-scenario, which results in 774 observations ( ix scenarios each for 129 subjects). ${ }^{9}$ We include subject fixed effects in all models to account for the interdependence of decisions across scenarios at the individual level.

The first column (Model 1) presents a baseline model of observed effort on the Nash predicted effort level. The coefficient on Nash effort level is positive and significant. Next we include an indicator for whether the subject is female in Model 2. The coefficient on Nash effort remains positive and significant while the coefficient on female is negative and significant. Although the women in our subject pool are slightly less risk averse than the male subjects, women choose significantly lower effort levels than male subjects. Model 3 includes the measure of risk aversion from the lottery choice experiment (mid-CRRA) and excludes gender. Here, we observe a negative and significant coefficient on mid-CRRA, providing some empirical evidence that risk aversion may be negatively associated with rent seeking behavior. The Nash coefficient remains positive and significant. ${ }^{10}$

\footnotetext{
${ }^{8}$ Millner and Pratt (1991) also demonstrate that the effect of risk aversion on rent seeking expenditures is dependent upon the structure of risk attitudes.

${ }^{9}$ Eleven subjects are omitted from this analysis. One individual was dropped due to missing information. Seven other subjects are dropped from the analysis because their effort choice is constant across scenarios. Three additional subjects are omitted from this analysis due to a missing value for "mid-CRRA" (missing values are generated when subjects make an irrational decision in the lottery choice experiment by choosing a certain low payoff over a certain high payoff).

${ }^{10}$ The difference in magnitudes of the coefficients $(0.577$ on the Nash prediction versus -258.8 on female and -448.6 on mid-CRRA) is due to the scale of the variables. The dependent variable (effort level) and Nash predicted effort level are measured on the same scale (hundreds), while the female variable is binary ( 0 or 1$)$ and the mid-CRRA variable takes on values between -0.55 and 1.37 .
} 
We next include both the female dummy and risk aversion parameter in Model 4, however the coefficient on mid-CRRA is no longer significant. This may be due to multicollinearity between gender and mid-CRRA. For this reason, we generate two interaction terms, Nash*Female and mid-CRRA*Female. Both interaction terms are included in the regression of Model 5. Here again, we see the coefficient on Nash effort is positive and significant at the $1 \%$ level. The coefficient on mid-CRRA is negative and significant, though of a smaller magnitude than in Model 3. The interaction terms are both negative and significant; the coefficient on the Nash*Female interaction term provides evidence that women are less affected by the Nash prediction than men. The negative and significant coefficient on the female risk aversion interaction term indicates that females choose lower levels of effort than men given the same level of risk aversion. Recall that theory is not clear on how risk aversion may affect effort choices; risk averse subjects may choose to keep as much of their starting budget as possible, or they may choose high effort levels to increase the probability of winning. Taken as a whole, our results suggest that risk aversion in general leads to lower effort choices and women prefer to keep more of their starting budget than men. 
Table 4: Regression Analysis of Observed Effort

\begin{tabular}{lccccc}
\hline & Model 1 & Model 2 & Model 3 & Model 4 & Model 5 \\
\hline Nash & $0.577^{* * *}$ & $0.577^{* * *}$ & $0.559^{* * *}$ & $0.559^{* * *}$ & $0.784^{* * *}$ \\
& $(5.87)$ & $(5.87)$ & $(5.58)$ & $(5.58)$ & $(6.12)$ \\
Female & & $-258.8^{* *}$ & & $-210.4^{* *}$ & \\
& & $(-2.08)$ & & $(-2.10)$ & \\
Mid-CRRA & & & $-448.6^{* * *}$ & -49.3 & $-194.8^{* * *}$ \\
& & & $(7.57)$ & $(-0.80)$ & $(-3.06)$ \\
Nash*Female & & & & $-0.477^{* *}$ \\
& & & & & $(-2.38)$ \\
Mid-CRRA*Female & & & & & $-253.8^{* * *}$ \\
& & & & & \\
& & & & & \\
$\mathrm{R}^{2}$ & 0.52 & 0.52 & 0.51 & 0.51 & 0.52 \\
\hline \hline
\end{tabular}

Notes: All models include individual fixed effects. t-statistics are in parentheses.

$*, * *$ and $* * *$ indicate significance at the $10 \%$ level, $5 \%$ level and $1 \%$ level, respectively.

\section{Discussion}

We experimentally test a rent seeking model with varying degrees of competition. Lower levels of competition imply that one subject's rent seeking expenditures have a smaller impact on the other subject's probability of winning a prize, and thus, expenditures by both subjects will be lower. Our results are consistent with previous findings that subjects overspend relative to the Nash prediction. We find some support for the theory that relaxing competition lowers the amount spent in the contest but not to the degree predicted by the model. This is consistent with Anderson and Stafford (2003) which found that expenditures moved in the direction predicted by theory when group size and entry fees changed, but overall expenditures exceeded the Nash prediction.

We contribute to the existing experimental research on rent seeking in two important ways. First, we study a model (Godwin et al. 2006) that allows for the possibility of multiple prizes by adding a variable competition parameter. With levels of competition lower than Tullock's (1980) standard model, both players can win the full amount of the prize. An 
important prediction of the model is that expenditures will fall with the level of competition. We are aware of only one other experimental study with multiple prizes. Shupp (2004) tested a model with a prize that was divisible among multiple winners but with a fixed pool of prize money across contests. This model also predicted lower expenditures as the prize was divided among more winners. Consistent with the findings of our study, Shupp (2004) reported that expenditures are lower when there are multiple prizes awarded. However, Shupp (2004) found that overall expenditure levels were lower than the Nash prediction.

Our second major contribution to the experimental rent seeking literature is a study of the behavioral effect of risk aversion on expenditures. We include a measure of risk aversion (Holt and Laury 2002) that has been validated in a number of other studies. Millner and Pratt (1991) controlled for risk aversion in an early experimental study by having subjects complete a lottery choice task that was similar to the Holt and Laury (2002) instrument. Based on the results of the task, they separated subjects into a "less risk averse" group and a "more risk averse" group. We build on this work by including a more precise measure of risk aversion (mid-CRRA) that allows subjects to be categorized across the full spectrum of risk preferences and provides a parameter that can be used in econometric analyses. Consistent with our findings, Millner and Pratt (1981) reported that expenditures were significantly higher among the less risk averse group. ${ }^{11}$ We also add to this work by identifying important interactions between gender and risk aversion in the context of a rent seeking experiment.

\section{Acknowledgements}

\footnotetext{
${ }^{11}$ Millner and Pratt (1981) also reported that expenditures in the more risk averse group were not significantly greater than the Nash prediction. Based on this finding, we separated subjects into three groups: risk loving, risk neutral, and risk averse. In each of the three groups, expenditures were significantly greater than the Nash prediction.
} 
This work was funded by the National Science Foundation (SES-0094800) and a William and Mary summer research grant. We thank Sarah Stafford for helpful suggestions on this project. 


\section{References}

Anderson, Lisa R. and Jennifer M. Mellor. (forthcoming). "Predicting Health Behaviors with an Experimental Measure of Risk Preference," Journal of Health Economics.

Anderson, Lisa R. and Sarah L. Stafford. 2003. "An Experimental Analysis of Rent Seeking Under Varying Competitive Conditions," Public Choice, 115: 199-216.

Berry, S. Keith. 1993. "Rent-Seeking with Multiple Winners," Public Choice, 77: 437-443.

Bullock, David S. and E. Elisabet Rutström. 2007. "Policy Making and Rent-Dissipation: An Experimental Test," Experimental Economics, 10:21-36.

Cadigan, John. 2007. “Two-Stage Team Rent-Seeking: Experimental Analysis," Southern Economic Journal, 74(1): 85-103.

Clark, Derek J. and Christian Riis. 1998. "Influence and the Discretionary Allocation of Several Prizes," European Journal of Political Economy, 14: 605-625.

Clark, Derek J. and Christian Riis. 1996. "A Multi-Winner Nested Rent-Seeking Contest," Public Choice, 87: 188-184.

Eckel, Catherine and Philip Grossman. (forthcoming). "Men, Women and Risk Aversion: Experimental Evidence," in Charles Plott and Vernon Smith, (eds.), Handbook of Experimental Economics Results, Volume 1. City: New York, North-Holland Publishing.

Godwin, R. Kenneth, Edward J. López and Barry J. Seldon. 2006. "Incorporating Policymaker Costs and Political Competition into Rent-Seeking Games," Southern Economic Journal, 73(1): 37-54.

Holt, Charles A. and Susan K. Laury. 2002. "Risk Aversion and Incentive Effects," The American Economic Review 92(5),1644-1655.

Millner, Edward L. and Michael D. Pratt. 1989. “An Experimental Investigation of Efficient Rent-Seeking," Public Choice, 62: 139-51.

Millner, Edward L. and Michael D. Pratt. 1991. "Risk Aversion and Rent-Seeking: An Extension and Some Experimental Evidence," Public Choice, 69: 81-92.

Öncüler, Ayse and Rachel Croson. 2005. "Rent-Seeking for a Risky Rent: A Model and Experimental Investigation,” Journal of Theoretical Politics, 17(4): 403-429.

Konrad, Kai A. and Harris Schlesinger. 1997. "Risk Aversion in Rent-Seeking and RentAugmenting Games," The Economic Journal, 107(445): 1671-1683. 
Shupp, Robert. 2004. "Single Versus Multiple Winner Rent-Seeking Contests: An Experimental Investigation," Working Paper.

Tullock, Gordon. 1980. "Efficient Rent Seeking," in Toward a Theory of the Rent-Seeking Society, Buchanan, Tollison and Tullock (eds), Texas A\&M Press, College Station, TX: 97-112.

Yates, Andrew J. and Jac. C. Heckelman. 2001. "Rent-Seeking in Multiple Winner Rent-Seeking Contests," European Journal of Political Economy, 17: 835-852. 


\section{Appendix A: Instructions}

This experiment is a study of individual behavior. The instructions are simple. If you follow them carefully and make good decisions you may earn a considerable amount of money, which will be paid to you privately, in cash, at the end of the experiment today. At this time, you will be paid $\$ 5$ for showing up for the experiment today.

You will be asked to make decisions under six different scenarios. You may make these decisions in any order you choose. After everyone has made decisions for all six scenarios, we will throw a 6-sided die to determine which scenario will be used to determine your earnings. If the throw of the die is 1 , we will use scenario 1 to determine your earnings. If the throw of the die is 2 , we will use scenario 2 to determine your earnings and so on.

Note that you will only be paid for one of the six scenarios, but no one will know which one until you have made decisions for all six. Therefore, you should think carefully about your decisions for all six possible scenarios since they are all equally likely to determine your earnings.

The basic structure of the six scenarios is the same. You will be randomly matched with one other person in this room. In each scenario, you will be matched with this same person but your partner's identity will not be revealed to you. In each pair there is an Orange player and a Blue player. The top of this sheet indicates whether you are the Orange or Blue player in your pair.

You start each scenario with $\$ 10$ in your spending account that you can use to compete for a prize worth an additional $\$ 10$. To compete for the $\$ 10$ prize you must choose an effort level between and including 0 and 1000. Each unit of effort costs $\$ 0.01$ which will be subtracted from your $\$ 10$ spending account. For example, if you choose an effort level of 1 , your spending account will be reduced to $\$ 9.99$. You may keep your entire $\$ 10$ spending account by choosing an effort level of 0 , but you will have no chance to win the prize. In that case, you will leave here today with $\$ 10$ plus the $\$ 5$ you already received for showing up for the experiment.

You cannot buy more effort than your spending account will allow (i.e. more than 1000 units of effort). Note that you have $\$ 10$ to spend in each scenario, since only one scenario will actually be used to determine your earnings. In other words, you do not have to spread your \$10 spending account over the six scenarios. Your cost of effort is the same across scenarios.

As stated earlier, you choose effort to compete for a prize of $\$ 10$. Your probability of winning the $\$ 10$ prize will depend on your effort, which we will call E, and your partner's effort, which we will call $P$. The probability you win the prize is calculated as E divided by the sum of (E plus $\alpha^{*} \mathrm{P}$ ) where $\alpha$ is an adjustment factor that determines how much your partner's effort level affects your probability of winning the prize. Smaller values of $\alpha$ indicate that your partner's effort level has a smaller effect on your probability of winning. You will be provided with an excel worksheet for each scenario that is preprogrammed with the correct probability function for that scenario. You can plug in sample values for your effort and your partner's effort and the worksheet will calculate your probability of winning the prize. Remember you will not know the actual effort level chosen by your partner when you make your decision. When you are ready to commit to an effort level for a particular scenario, you should record it on the Record Sheet you have been given.

Once a scenario is chosen for payment using the throw of the 6-sided die, we will enter your effort choice and your partner's effort choice into the appropriate worksheet to determine your probability of winning the prize. Then we will use pokers chips to determine whether or not you win the prize based on your probability. We will place 100 poker chips in an opaque container. Each poker chip has an Orange 
number on one side and a Blue number on the other side. The numbers range from 1 to 100 and the sum of the Blue number and the Orange number on each poker chip is 100. For example, if the Blue number on the chip is 25 , the Orange number is 75 . Below we explain specifically how we will make random draws from this bag of poker chips to determine whether or not you win the prize.

As noted above your probability of winning the prize depends on your effort (E), your partner's effort (P), and an adjustment factor, $\alpha$ such that your probability $=\mathrm{E} /\left[\mathrm{E}+\alpha^{*} \mathrm{P}\right]$.

In Scenario 1 and Scenario 2, the adjustment factor is 1 , so the probability you win the prize is equal to your share $(E)$ of the total effort chosen by you and your partner $(E+P)$. For example, if you and your partner choose the same effort, you will each have a 50\% chance of winning the $\$ 10$ prize.

In scenario 1, only one person in each pair will win the prize. If this scenario is chosen for payment, we will draw one of the numbered poker chips from the container to determine who wins the prize. If you are the Orange player, you win the prize if the Orange number on the poker chip is less than or equal to your probability of winning (calculated from the excel worksheet). If you are the Blue player, you win the prize if the Blue number on the poker chip is less than or equal to your probability. Since only one of the two players in each pair can win the $\$ 10$ prize in this scenario, we will flip the poker chip to determine whether the Orange or Blue player wins the prize in the event of a tie.

In scenario 2, there are four possible outcomes: (1) both you and your partner win a $\$ 10$ prize; (2) you win a $\$ 10$ prize and your partner does not win; (3) your partner wins a $\$ 10$ prize and you do not win or (4) no one wins the prize. If Scenario 2 is chosen for payment, we will draw two numbered poker chips. First we will draw for the Blue player. If the Blue number on the chip is less than or equal to the Blue player's probability, the Blue player will win a $\$ 10$ prize. We will then return the chip to the container and we will draw a chip for the Orange player. If the Orange number on that chip is less than or equal to the Orange player's probability, the Orange player will win a $\$ 10$ prize.

In Scenario 3 through Scenario 6, the adjustment factor is less than 1, so your partner's effort level is less important in determining your probability of winning in these scenarios than in Scenario 1 and Scenario 2. In Scenario 3, the adjustment factor is 0.50 so the probability you win the prize is E divided by E plus $0.50 * \mathrm{P}$. For example, if you and your partner choose the same effort level in this scenario, you will each have a $67 \%$ chance of winning the prize. In Scenario 4, the adjustment factor is 0.25 . In Scenario 5, the adjustment factor is 0.10 and in Scenario 6, the adjustment factor is 0.05. As in Scenario 2, there are four possible outcomes in Scenarios 3 through 6: (1) both you and your partner win a \$10 prize; (2) you win a $\$ 10$ prize and your partner does not win; (3) your partner wins a $\$ 10$ prize and you do not win or (4) no one wins the prize. If one of these scenarios is chosen for payment, we will draw two numbered poker chips. First we will draw for the Blue player. If the Blue number on the chip is less than or equal to the Blue player's probability, the Blue player will win a $\$ 10$ prize. We will then return the chip to the container and we will draw a chip for the Orange player. If the Orange number on that chip is less than or equal to the Orange player's probability, the Orange player will win a $\$ 10$ prize.

As noted above, you will be provided with an excel worksheet to calculate sample probabilities for every scenario. Turn your attention to the computer screen which is currently set on Scenario 1. You may change scenarios by clicking on the tabs labeled Scenario1, Scenario2, etc. on the bottom/left side of the screen. Notice that each worksheet shows the scenario number at the top of the screen. The relevant adjustment factor and probability formula for each scenario are listed under the scenario number. You make sample calculations by entering effort choices in the green and gold boxes. You should enter YOUR sample effort choice in the green box and YOUR PARTNER'S sample effort choice in the gold 
box for each scenario. Notice that these effort levels are initially set at zero which results in a zero probability of you winning a prize, which is displayed in the red box. At this point you should change the sample effort levels for you and your partner and notice how your probability of winning a prize changes.

\section{SUMMARY}

You have already been randomly paired with a partner. You begin by choosing an effort level between 0 and 1000 for each of 6 scenarios. Effort costs $\$ 0.01$ per unit and you can spend up to $\$ 10$ on effort in each scenario. The scenarios vary in terms how your partner's effort level affects your probability of winning a $\$ 10$ prize and whether or not both you and your partner may win a $\$ 10$ prize. For each scenario, you have an excel worksheet to calculate your probability of winning a prize for sample combinations of effort levels.

Once everyone has chosen their effort levels for each scenario, we will select the scenario for which you will be paid using the 6-sided die. Then we will use the appropriate excel worksheet for that scenario to determine your probability of winning given your effort choice and the actual effort choice of your partner. For each pair, we will determine the winner or winners by drawing poker chips from an opaque container. If you are a winner, you receive a $\$ 10$ prize plus the remaining balance in your spending account ( $\$ 10$ minus the cost of your effort level) in addition to the $\$ 5$ you received for showing up for the experiment. If you are not a winner you receive the remaining balance in your spending account $(\$ 10$ minus the cost of your effort level) in addition to the $\$ 5$ you received for showing up for the experiment.

Please use the excel worksheets on your computer screen to make sample calculations for each scenario. When you are ready to choose your effort level for a scenario write it on the Record Sheet you were provided. It is important that you write your decision on your Record Sheet because we will not save the excel worksheets you use to make sample calculations. Be careful not to let others see what you write on your Record Sheet. This information should be private. Anyone caught trying to look at someone else's Record Sheet will be asked to leave the experiment and will not be paid. Once you have made all of your decisions, turn over your sheet and wait quietly until everyone has recorded their decisions. If you have a question, raise your hand and we will come to your desk to answer it. 


\section{Appendix B: Screen Shots of the Excel Worksheets Provided to Subjects}
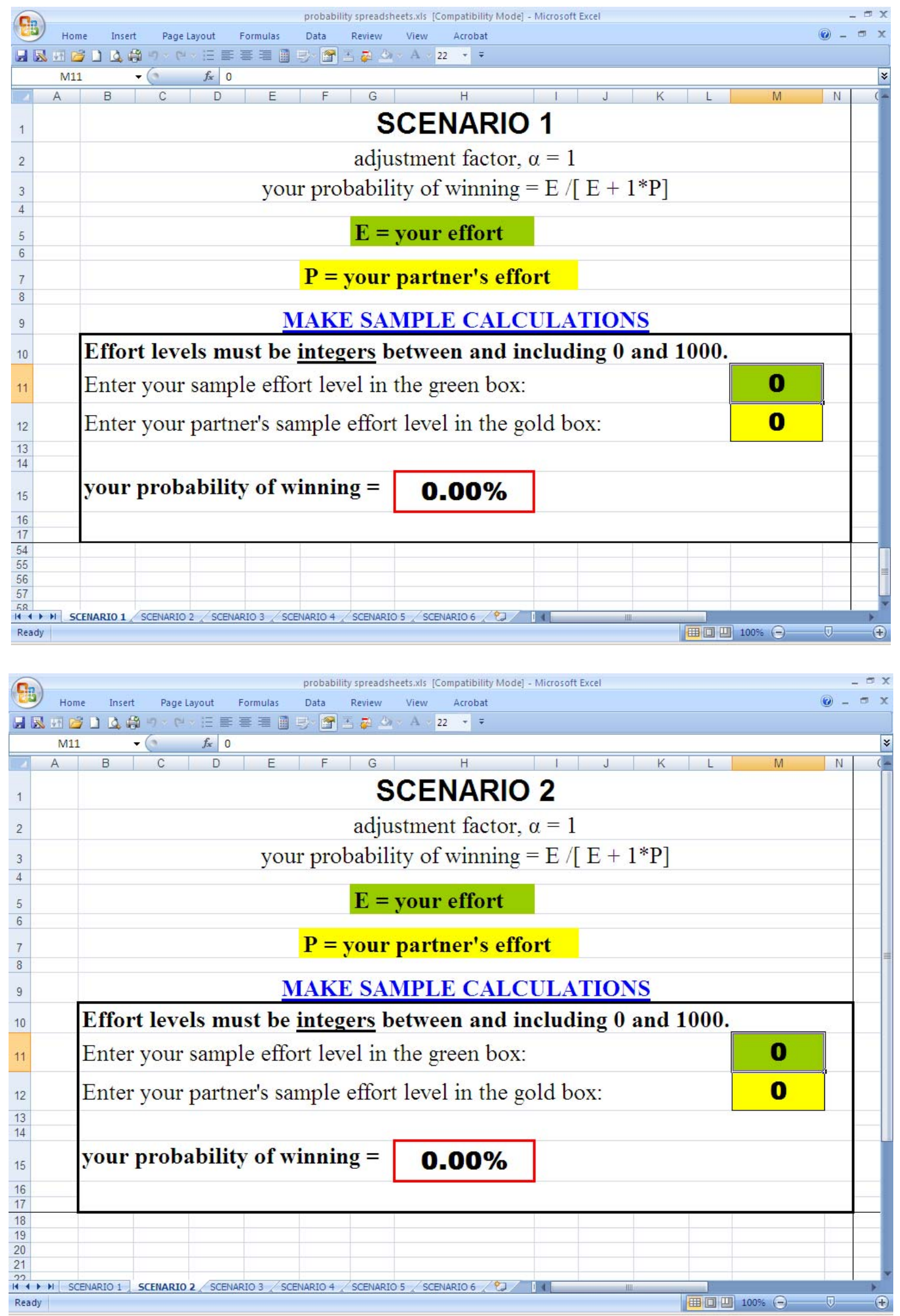

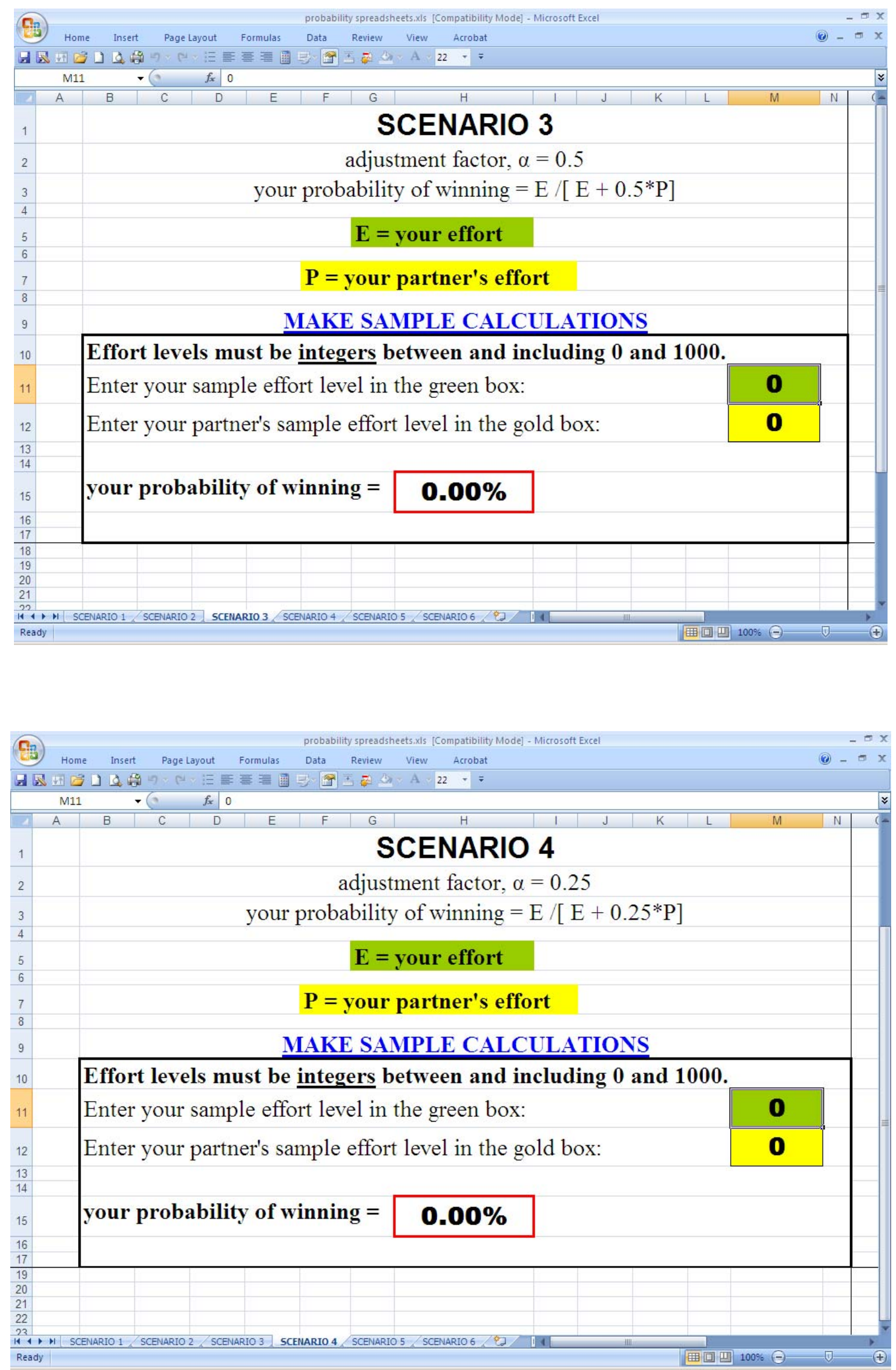

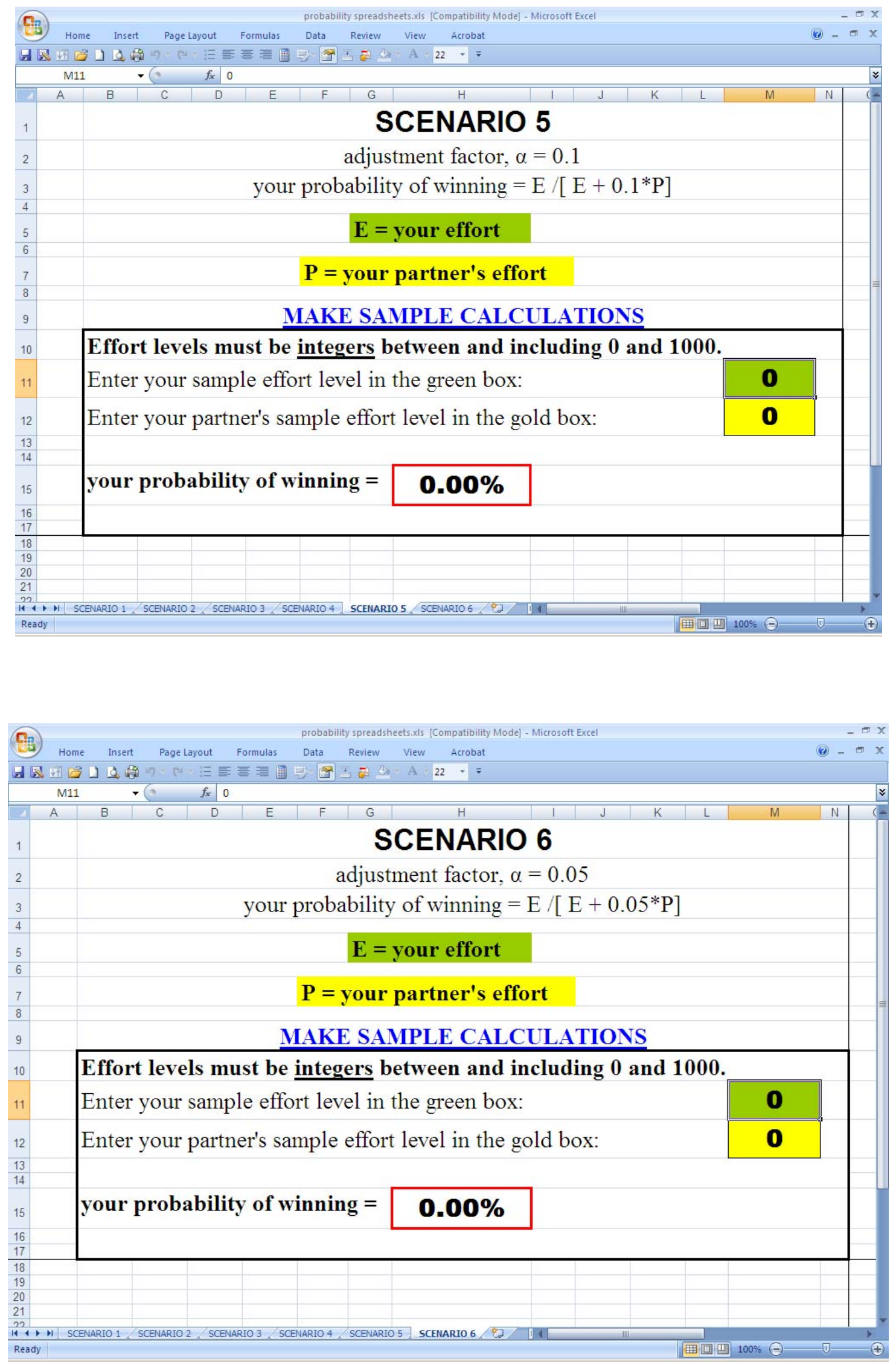